\title{
Efficacy and Safety Evaluation of Transurethral Resection of the Prostate versus Plasmakinetic Enucleation of the Prostate in the Treatment of Massive Benign Prostatic Hyperplasia
}

\author{
Jian Zhang ${ }^{a}$ Yonghui Wang ${ }^{b}$ Shuang Li $^{b}$ Shipeng Jin ${ }^{b}$ Shiqing Zhang ${ }^{a}$ \\ Chunli Zhao ${ }^{c}$ Wenzeng Yang ${ }^{c}$ Shujin Cuid Yuexin Liu ${ }^{b}$ \\ aDepartment of Urology, Beijing Geriatric Hospital, Beijing, China; ${ }^{b}$ Department of Urology, Beijing TongRen \\ Hospital, Capital Medical University, Beijing, China; 'Department of Urology, Affiliated Hospital of Hebei University, \\ Baoding, China; dDepartment of Urology, Beijing Luhe Hospital, Capital Medical University, Beijing, China
}

\section{Keywords \\ Benign prostatic hyperplasia - International Index of Erectile Function · International prostate symptom score · Transurethral bipolar plasmakinetic enucleation of the prostate $\cdot$ Transurethral resection of the prostate}

\begin{abstract}
Background: Benign prostatic hyperplasia (BPH) is a common chronic progressive disease resulting in urinary obstruction in aging men. It comes to more and more patients with massive BPH with the aging of society and extension of life expectancy. Objective: The aim of the study was to compare the clinical efficacy, safety, and complications between transurethral bipolar plasmakinetic enucleation of the prostate (PKEP) and transurethral resection of the prostate (TURP) in the treatment of massive BPH. Design and Setting: Patients with BPH were divided into the PKEP group and the TURP group randomly. Intraoperative blood loss (BL), operation time (OT), resected tissue weight (RTW), gland resection ratio (GRR), postoperative indwelling ureter time (IUT), bladder fistula time (BFT) and hospital stay time (HST), preoperative and
\end{abstract}

Jian Zhang and Yonghui Wang contributed equally to this work.

$\begin{aligned} & \text { karger@karger.com } \\ & \text { www.karger.com/uin }\end{aligned}$
Karger $\%$

postoperative serum sodium concentration (SSC), hemoglobin concentration (HGB), prostate weight (PW), postvoid residual (PVR), maximum urinary flow rate $\left(Q_{\max }\right)$, international prostate symptom score (IPSS), quality of life (QOL), International Index of Erectile Function (IIEF), and other complications were analyzed and compared respectively. Results: There was no statistical difference in preoperative IPSS, preoperative $\mathrm{QOL}$ score, preoperative PVR, preoperative $Q_{\max }$ postoperative QOL score, postoperative PVR, postoperative $Q_{\max }$ IPSS difference value (DV), $Q_{\max } \mathrm{DV}$, and PVR DV between the PKEP group and the TURP group ( $p>0.05)$. OT, BL, IUT, BFT, HST, and postoperative IPSS in the PKEP group were significantly lower than that in the TURP group $(p<0.01)$. RTW and GRR in the PKEP group were significantly higher than that in the TURP group $(p<0.01)$. QOL DV in the PKEP group was higher than that in the TURP group $(p<0.05)$. There was statistical difference in SSC DV between the PKEP group and the TURP group $(p<0.05)$. There was significant statistical difference in postoperative $\mathrm{PW}$, postoperative HGB, PW DV, and HGB DV between the PKEP group and the TURP group $(p<0.01)$. There was significant statistical difference in IPSS, QOL, PVR, and $Q_{\max }$ between postoperative val- 
ue and preoperative value in both groups $(p<0.01)$. The incidence of transurethral resection syndrome, obturator nerve reflex, transient urinary incontinence, and retrograde ejaculation between the PKEP group and the TURP group has no statistical difference $(p>0.05)$. Capsule perforation, blood transfusion, secondary hemorrhage, bladder neck contracture, and urethral stricture in the PKEP group were lower than that in the TURP group $(p<0.05)$. Bladder spasm in the PKEP group was significantly lower than that in the TURP group $(p<0.01)$. There was no statistical difference in preoperative and postoperative IIEF-5, effective erectile frequency, telotism average tension, sustainable telotism average time, and sexual dissatisfaction between the PKEP group and the TURP group ( $p>0.05$ ). Conclusions: PKEP and TURP have similar clinical efficacy in the treatment of massive BPH. PKEP has advantages in shorter $\mathrm{OT}$, less $\mathrm{BL}$, more GRR, and fewer complications, but the long-term therapeutic effect of PKEP needs further follow-up.

(c) 2021 S. Karger AG, Basel

\section{Introduction}

Benign prostatic hyperplasia (BPH) is a common chronic progressive disease resulting in the enlargement of the prostate gland and bladder outlet obstruction in aging men [1]. With the aging of society and extension of life expectancy, more and more patients are diagnosed with massive BPH [2]. Transurethral resection of the prostate (TURP) has ever been known as the "gold standard" for BPH treatment. TURP still has certain restrictions such as bleeding, prostatic volume, transurethral resection syndrome (TURS), and so on [3, 4]. Recently, transurethral bipolar plasmakinetic enucleation of the prostate (PKEP) has been introduced as the new method for massive BPH treatment. On the basis of TURP and suprapubic prostatectomy, PKEP was introduced to overcome the shortcomings of TURP [5]. The study compared the clinical efficacy, safety, and complications of PKEP with those of TURP in patients with massive BPH.

\section{Materials and Methods}

\section{The Clinical Data}

All enrolled patients gave written informed consent. We analyzed the clinical data for 99 patients who underwent TURP and 101 patients who underwent PKEP for BPH from July 2014 to July 2016 at our center retrospectively. The inclusion criteria included patients who had progressive urination dysfunction and poor efficacy with conservative medical treatment for more than 1 year by international prostate symptom score (IPSS) and quality of life (QOL) evaluation. Urodynamic examination revealed severe bladder outlet obstruction with maximum flow rate $\left(Q_{\max }\right)<10 \mathrm{~mL} / \mathrm{s}$, good bladder detrusor tolerableness, and residual urine volume (postvoid residual $[\mathrm{PVR}]$ ) $\geq 50 \mathrm{~mL}$. Massive $\mathrm{BPH}$ was considered by digital rectal examination, ultrasound examination (prostate gland larger than $80 \mathrm{~g}$ ), and postoperation pathology. The International Index of Erectile Function-5 (IIEF-5) score was $\geq 12$. Patients had normal sex partners in the follow-up period. The exclusion criteria were bladder tumors, bladder diverticulum, neurogenic bladder, weak detrusor, serious urethral stricture (US), serious medical diseases, and long-term therapy drugs affecting sexual function. Patients were divided into the PKEP group and the TURP group according to the random principle. For $101 \mathrm{pa}-$ tients aged 62 years to 71 years (mean $66 \pm 5.5$ years), prostatespecific antigen (PSA) was $1.5 \pm 0.8 \mathrm{ng} / \mathrm{mL}$ in the PKEP group. For 99 patients aged 62 years to 70 years (mean $65 \pm 5.0$ years), PSA was $1.7 \pm 0.7 \mathrm{ng} / \mathrm{mL}$ in the TURP group.

\section{Surgery and Related Data Analysis Methods}

Surgical Methods

Patients took continuous epidural anesthesia, bladder lithotomy position, bladder puncture ostomy, and low-pressure bladder irrigation. In the TURP group, the surgeon needed to observe the condition of the urethra, verumontanum, the middle and the bilateral lobes of the prostate, and the hyperplastic degree of every lobe through the resectoscope under video-assisted endosurgical system guidance. To identify the anatomical landmarks such as ureteral orifices, verumontanum, and external sphincter of the urethra, the middle lobe, lateral lobe, and anterior junction were resected in order. In the process of resection, the hyperplastic tissue was resected layer by layer, and the bleeding point was dealt with after each resection. In the process of resection, the first priority was to accurately identify the anatomical markers mentioned above and not blindly remove any of them. After tissue resection, resectoscope examination was performed to make sure that there was no bleeding and residual tissue mass. Then, continuous bladder irrigation was carried out until hematuria sufficiently resolved.

In the PKEP group, the operator incised the urethral mucosa circularly deep to the surgical capsule above the verumontanum. Then, the operator enucleated and pushed the mid lobe from the distal mucosa toward the bladder neck. Further, the operator used the loop to cut off the tissue between the lobe and the surgical capsule and then inserted the tip into the previous cleavage to detach the adenoma along with the capsule. The hemorrhage spots on the capsule surface were identified and coagulated. This procedure progressed toward the bladder neck until the circular fiber of the bladder neck was identified. Then, the bilateral lobes along the surgical capsule were detached clockwise or anticlockwise from the 5 or the 7 o'clock position of the prostatic apex to the 12 o'clock position. At the point, most of the blood supply to the lobes was blocked. The adenoma was resected rapidly and thoroughly by the loop electrode without serious hemorrhage. When resection was completed, the surgeon needed to use the resection loop and mirror sheath to push the excised tissue to the bladder as the whole and ground tissue in the bladder. Then, continuous bladder irrigation until hematuria sufficiently resolved.

Data Analysis

Intraoperative blood loss (BL), resected tissue weight (RTW), gland resection ratio (GRR), operation time (OT), the postopera- 
Table 1. OT, BL, RTW, GRR, IUT, BFT, and HST comparison

\begin{tabular}{lrlllllll}
\hline Group & $N$ & OT, min & BL, mL & RTW, g & GRR & IUT, h & BFT, h & HST, days \\
\hline TURP & 99 & $106.95 \pm 15.80$ & $230.62 \pm 90.85$ & $62.65 \pm 14.86$ & $0.65 \pm 0.08$ & $66.95 \pm 19.60$ & $41.63 \pm 6.07$ & $4.82 \pm 0.55$ \\
PKEP & 101 & $89.04 \pm 14.66^{\# \#}$ & $120.12 \pm 54.27^{\# \#}$ & $78.79 \pm 14.70^{\# \#}$ & $0.76 \pm 0.06^{\# \#}$ & $41.60 \pm 12.07^{\# \#}$ & $16.49 \pm 3.31^{\# \#}$ & $3.27 \pm 0.46^{\# \#}$ \\
\hline$t$ & & 8.313 & 10.466 & 7.723 & 11.911 & 10.985 & 36.279 & 21.638 \\
\hline$p$ value & 0.000 & 0.000 & 0.000 & 0.000 & 0.000 & 0.000 & 0.000 \\
\hline
\end{tabular}

OT, operation time; BL, blood loss; RTW, resected tissue weight; GRR, gland resection ratio; IUT, indwelling ureter time; BFT, bladder fistula time; HST, hospital stay time; TURP, transurethral resection of the prostate; PKEP, plasmakinetic enucleation of the prostate. ${ }^{\#}$ Compared with the TURP group, $p<0.01$.

Table 2. Preoperative and postoperative PW, HGB, and SSC comparison

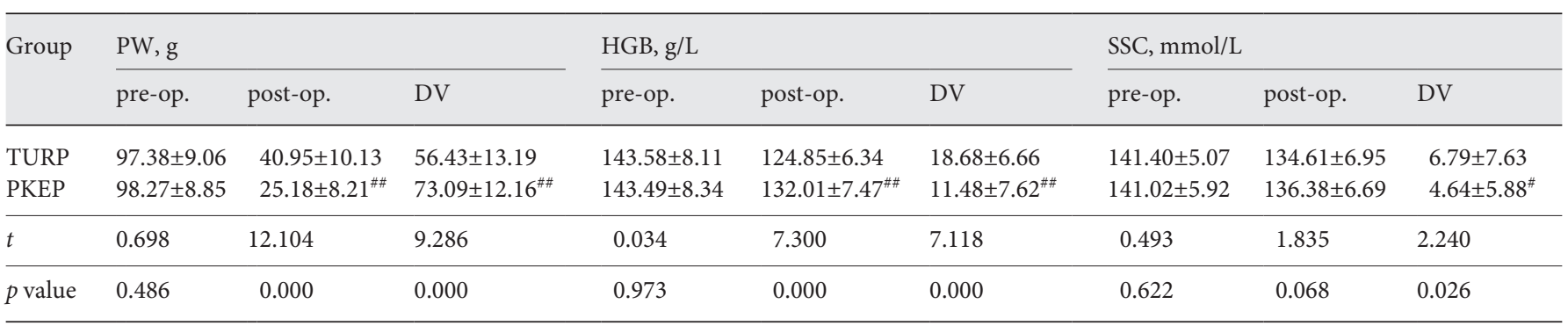

PW, prostate weight; HGB, hemoglobin concentration; SSC, serum sodium concentration; DV, difference value; pre-op, preoperative; post-op, postoperative. ${ }^{\#}$ Compared with the TURP group, $p<0.05 .{ }^{\#}$ Compared with the TURP group, $p<0.01$.

tive indwelling ureter time (IUT), bladder fistula time, hospital stay time (HST), TURS, obturator nerve reflex (ONR), capsule perforation (CP), blood transfusion (BT), secondary hemorrhage $(\mathrm{SH})$, bladder spasm (BS), retrograde ejaculation (RE), transient urinary incontinence (TUI), sexual dissatisfaction, bladder neck contracture (BNC), and US incidence were analyzed retrospectively. Preoperative and postoperative serum sodium concentration (SSC), hemoglobin concentration (HGB), prostate weight (PW), residual urine $(\mathrm{PVR})$, maximum urinary flow rate $\left(Q_{\max }\right)$, IPSS, quality of life (QOL) score, IIEF-5 score, nocturnal penile tumescence combined with polysomongraphy were also analyzed.

Intraoperative $\mathrm{BL}$ was measured with the following formula: $\mathrm{BL}$ volume $(\mathrm{mL})=($ Erythrocyte concentration in urine routine result of an intraoperative mixed sample of bladder perfusion fluid $x$ $10^{9} / \mathrm{L} \times$ bladder perfusion fluid volume $\times \mathrm{L}$ )/Erythrocyte concentration $(/ \mathrm{L})$ in blood routine result of the preoperative blood sample. HGB and SSC were detected $30 \mathrm{~min}$ before and after the surgery. PW was measured by transverse diameter $\times$ anteroposterior diameter $\times$ longitudinal diameter $\times 0.52 \times 1.05$ under ultrasound. Excision tissue was weighed as RTW under electronic scales. GRR was RTW/preoperative PW. PVR was detected by catheterization assay; $Q_{\max }$, IPSS, QOL, and IIEF-5 score were compared with those obtained 24 months postoperatively. RE refers to be without semen ejaculation during sexual intercourse and to discover sperm and fructose in urine after intercourse submission. IIEF-5 score and other data were recorded with questionnaires and other forms.

TURP versus PKEP in the Treatment of Massive BPH

\section{Statistical Analysis}

SPSS 13.0 software package was used. Measurement data were expressed as $\bar{x} \pm$ SD. Statistical difference analysis was carried out using $t$ test, $\chi^{2}$ test, or Fisher exact test. $p<0.05$ was considered as statistical difference. $p<0.01$ was considered as significant statistical difference.

\section{Results}

\section{Comparison of Clinical Parameters}

OT, BL, IUT, BFT, and HST in the PKEP group were significantly lower than those in the TURP group $(p<$ $0.01)$. RTW and GRR in the PKEP group were significantly higher than those in the TURP group $(p<0.01)$ (Table 1).

There was no statistical difference in preoperative PW, preoperative $\mathrm{HGB}$, preoperative SSC, and postoperative SSC between the PKEP and TURP groups $(p>0.05)$. There was statistical difference in preoperative and postoperative SSC and in SSC difference value (DV) between the PKEP and TURP groups $(p<0.05)$. There was sig- 
Table 3. Postoperative and preoperative $Q_{\max }$ and IPSS comparison

\begin{tabular}{|c|c|c|c|c|c|c|}
\hline \multirow[t]{2}{*}{ Group } & \multicolumn{3}{|l|}{ IPSS } & \multicolumn{3}{|l|}{ QOL score } \\
\hline & pre-op. & post-op. & $\mathrm{DV}$ & pre-op. & post-op. & DV \\
\hline TURP & $26.36 \pm 4.09$ & $5.06 \pm 1.48^{* *}$ & $21.30 \pm 4.14$ & $4.49 \pm 1.10$ & $1.76 \pm 0.78^{* *}$ & $2.74 \pm 1.44$ \\
\hline PKEP & $26.24 \pm 3.59$ & $4.13 \pm 1.39^{\# \#, * *}$ & $22.11 \pm 3.37$ & $4.59 \pm 1.07$ & $1.57 \pm 0.74^{* *}$ & $3.02 \pm 1.22^{\#}$ \\
\hline$t$ & 0.231 & 4.585 & 1.507 & 0.646 & 1.702 & 1.500 \\
\hline$p$ value & 0.817 & 0.000 & 0.133 & 0.519 & 0.090 & 0.0135 \\
\hline
\end{tabular}

$Q_{\max }$, maximum urinary flow rate; IPSS, international prostate symptom score; QOL, quality of life; DV, difference value; pre-op, preoperative; post-op, postoperative; TURP, transurethral resection of the prostate; PKEP, plasmakinetic enucleation of the prostate. ${ }^{\#}$ Compared with the TURP group, $p<0.05$. \#\# Compared with the TURP group, $p<0.01$. $^{* *}$ Compared with preoperation, $p<0.01$.

Table 4. Postoperative and preoperative PVR, $Q_{\max }$, IPSS, and QOL comparison

\begin{tabular}{|c|c|c|c|c|c|c|}
\hline \multirow[t]{2}{*}{ Group } & \multicolumn{3}{|l|}{ PVR, mL } & \multicolumn{3}{|l|}{$Q_{\max }, \mathrm{mL} / \mathrm{s}$} \\
\hline & pre-op. & post-op. & DV & pre-op. & post-op. & DV \\
\hline TURP & $69.45 \pm 12.57$ & $17.65 \pm 6.07^{* *}$ & $51.81 \pm 12.27$ & $7.66 \pm 1.63$ & $15.45 \pm 3.26^{* *}$ & $7.80 \pm 3.11$ \\
\hline PKEP & $70.32 \pm 10.89$ & $16.46 \pm 5.36^{* *}$ & $53.86 \pm 11.37^{\#}$ & $7.45 \pm 1.78$ & $16.01 \pm 4.16^{* *}$ & $8.56 \pm 3.89^{\# \#}$ \\
\hline$t$ & 0.519 & 1.471 & 1.228 & 0.866 & 1.050 & 1.534 \\
\hline$p$ value & 0.605 & 0.143 & 0.221 & 0.387 & 0.29 & 0.127 \\
\hline
\end{tabular}

PVR, postvoid residual; $Q_{\max }$, maximum urinary flow rate; DV, difference value; pre-op, preoperative; postop, postoperative; TURP, transurethral resection of the prostate; PKEP, plasmakinetic enucleation of the prostate. \# Compared with the TURP group, $p<0.05$. \# Compared with the TURP group, $p<0.01$. ** Compared with preoperation, $p<0.01$.

nificant statistical difference in postoperative PW, postoperative HGB, PW DV, and HGB DV between the PKEP and TURP groups $(p<0.01)$ (Table 2$)$.

\section{Comparison of Clinical Follow-Up Indicators}

There was no statistical difference in preoperative IPSS, preoperative QOL score, preoperative PVR, preoperative $Q_{\max }$, postoperative QOL score, postoperative PVR, postoperative $Q_{\max }$ IPSS, DV, $Q_{\max } \mathrm{DV}$, and PVR DV between the PKEP and TURP groups $(p>0.05)$. QOL DV in the PKEP group was higher than that in the TURP group $(p<0.05)$. Postoperative IPSS in the PKEP group was significantly lower than that in the TURP group $(p<$ 0.01 ). There was significant statistical difference in IPSS, QOL, PVR, and $Q_{\max }$ between postoperative value and preoperative value $(p<0.01)$ ( $T$ was $44.259,17.155$, 30.672 , and 24.706, respectively, in the TURP group. $T$ was $61.618,23.849,36.700$, and 23.604 , respectively, in the PKEP group) (Tables 3, 4).
There was no statistical difference in TURS, ONR, TUI, and RE incidence between the PKEP and TURP groups ( $p>0.05)$. CP, BT, SH, BNC, and US in the PKEP group were lower than those in the TURP group $(p<$ $0.05)$. BS in the PKEP group was significantly lower than that in the TURP group $(p<0.01)$ (Table 5).

\section{Comparison of Sexual Function and Related Index}

There was no statistical difference in sexual dissatisfaction between the PKEP and TURP groups $(p>0.05)$ (Table 5). There was no statistical difference in preoperative and postoperative IIEF-5, EEF, TAT, and STAT between the PKEP and TURP groups $(p>0.05)$. There was no statistical difference in IIEF- 5 between postoperative value and preoperative value $(p>0.05)$ ( $p$ and $t$ were 0.362 and 0.917 , respectively, in the TURP group; $p$ and $t$ were 0.056 and 1.937, respectively, in the PKEP group). There was no statistical difference in EEF between postoperative value and preoperative value $(p>0.05)$ ( $p$ and $t$ were 0.256 and 
Table 5. Major complication incidence comparison between the PKEP and TURP groups (number, 100\%)

\begin{tabular}{lrllllllllll}
\hline Group & $N$ & TURS & ONR & CP & BT & SH & BS & TUI & SLD & RE & BNC and US \\
\hline TURP, $n(\%)$ & 99 & $3(3.0)$ & $5(5.1)$ & $7(7.1)$ & $7(7.1)$ & $8(8.1)$ & $28(28.3)$ & $20(20.2)$ & $31(31.3)$ & $16(16.2)$ & $9(9.1)$ \\
PKEP, $n(\%)$ & 101 & $0(0)$ & $1(1.0)$ & $1(1.0)^{\#}$ & $1(1.0)^{\#}$ & $1(1.0)^{\#}$ & $13(12.9)^{\# \#}$ & $24(23.8)$ & $21(41.3)$ & $26(25.7)$ & $2(4.3)^{\#}$ \\
\hline$\chi^{2}$ & & & & & & & 7.286 & 0.369 & 2.876 & 2.766 & 4.864 \\
\hline$p$ value & & 0.119 & 0.117 & 0.034 & 0.034 & 0.018 & 0.007 & 0.543 & 0.090 & 0.096 & 0.027 \\
\hline
\end{tabular}

TURP, transurethral resection of the prostate; PKEP, plasmakinetic enucleation of the prostate; TURS, transurethral resection syndrome; ONR, obturator nerve reflex; CP, capsule perforation; BT, blood transfusion; SH, secondary hemorrhage; BS, bladder spasm; TUI, transient urinary incontinence; SLD, sexual dissatisfaction; RE, retrograde ejaculation; BNC, bladder neck contracture; US, urethral stricture. ${ }^{\#}$ Compared with the TURP group, $p<0.05$. ${ }^{\# \#}$ Compared with the TURP group, $p<0.01$.

Table 6. Preoperative and postoperative IIEF-5 score and NPT index comparison

\begin{tabular}{|c|c|c|c|c|c|c|c|c|}
\hline \multirow[t]{2}{*}{ Group } & \multicolumn{2}{|l|}{ IIEF-5 score } & \multicolumn{2}{|l|}{ EEF, times } & \multicolumn{2}{|l|}{ TAT, g } & \multicolumn{2}{|l|}{ STAT, s } \\
\hline & pre-op. & post-op. & pre-op. & post-op & pre-op. & post-op. & pre-op. & post-op. \\
\hline TURP & $17.42 \pm 3.24$ & $17.03 \pm 2.59$ & $4.01 \pm 0.93$ & $3.90 \pm 0.85$ & $108.51 \pm 16.27$ & $106.98 \pm 15.72$ & $509.90 \pm 167.53$ & $487.88 \pm 168.55$ \\
\hline PKEP & $17.62 \pm 2.98$ & $17.00 \pm 2.78$ & $4.00 \pm 0.93$ & $3.75 \pm 1.04$ & $109.73 \pm 17.59$ & $108.03 \pm 16.41$ & $504.01 \pm 163.39$ & $483.47 \pm 159.72$ \\
\hline$t$ & 0.454 & 0.080 & 0.152 & 1.089 & 0.719 & 0.462 & 0.252 & 0.190 \\
\hline$p$ value & 0.650 & 0.936 & 0.880 & 0.277 & 0.473 & 0.645 & 0.802 & 0.849 \\
\hline
\end{tabular}

TURP, transurethral resection of the prostate; PKEP, plasmakinetic enucleation of the prostate; IIEF-5, International Index of Erectile Function-5; EEF, effective erectile frequency; TAT, telotism average tension; STAT, sustainable telotism average time; pre-op, preoperative; post-op, postoperative; NPT, nocturnal penile tumescence combined with polysomongraphy.

1.142 , respectively, in the TURP group; $p$ and $t$ were 0.053 and 1.961, respectively, in the PKEP group). There was no statistical difference in TAT between postoperative value and preoperative value $(p>0.05)$ ( $p$ and $t$ were 0.626 and 0.489 , respectively, in the TURP group; $p$ and $t$ were 0.446 and 0.765 , respectively, in the PKEP group.) There was no statistical difference in STAT between postoperative value and preoperative value $(p>0.05)$ ( $p$ and $t$ were 0.346 and 0.946 , respectively, in the TURP group; $p$ and $t$ were 0.399 and 0.847 , respectively, in the PKEP group) (Table 6).

\section{Discussion}

The prevalence of $\mathrm{BPH}$ has increased with the development of social economy and the aging of society in recent decades. The incidence rate of $\mathrm{BPH}$ is over $50 \%$ in the male population aged over 60 years and more than $80 \%$ in men aged over 90 years $[1,6]$. Symptoms secondary to bladder outlet obstruction include lower urinary

TURP versus PKEP in the Treatment of Massive BPH tract symptoms, renal insufficiency, hematuria, recurrent urinary tract infection, and bladder calculi seriously affecting the QOL of BPH patients. Surgical treatment is the preferred treatment for poor drug treatment efficiency. The most commonly used surgical method is TURP, which has ever been considered the gold standard [7-9]. $\mathrm{BPH}$ is treated with TURP with high-frequency electric and heat energy. TURP has shortcomings, such as perioperative hemoglobin loss, short circuit risk of monopolar transurethral resection loop and mirror sheath, heavy damage with heat penetration, slow healing, and infection under the scab. TURP also has difficulty to completely remove massive hyperplasia tissue, particularly in the prostate apex, which can cause incontinence, ED, and $\mathrm{BPH}$ recurrence [10]. TURP is prone to excise too much local tissue, which can lead to $\mathrm{CP}$ and severe venous bleeding. If the excision scope cannot extend to the surgical capsule fibers, TURP may lead to residual proliferative tissue and postoperative recurrence [11].

TURP has the above shortcomings; therefore, PKEP has been promoted as the alternative surgical way. PKEP 
is the third-generation transurethral treatment of $\mathrm{BPH}$ with advanced equipment and technology based on TURP and transurethral vaporization $[12,13]$. The bipolar electric cutting system was applied in clinical use in 1995 by Peter Gilling. The enucleation process started at the bladder neck. The surgeon needed to resect bladder neck incisions at the 5 and 7 o'clock positions down to the surgical capsule first. Then, the surgeon enucleated the median lobe and the lateral lobes, and made the lobes float into the bladder separately. In the end, the surgeon incised the bladder neck at the 12 o'clock position on each side, and then the superior aspect of the lateral lobe was gradually peeled off the capsule [14]. This procedure was improved by $\mathrm{Xu}$ et al. [15], who used this procedure to treat $\mathrm{BPH}$ in China. It includes several special steps. First, operators needed to cut the short horizontal incision at 6 o'clock, in order to expose the surgical capsule of the prostate apex. Second, the operators dug the tunnel proximally to the bladder neck beneath the median lobe and along with the surgical capsule. After digging a tunnel through the verumontanum to the bladder neck, the operators enucleated the right lateral lobe in the clockwise direction and enucleated the left lateral lobe in the anticlockwise direction [15].

We have improved the surgical method of BPH treatment based on the two surgical procedures. PKEP combines the advantages of open prostatectomy and TURP: hyperplasia glands were conversely enucleated with blunt or sharp dissection along the middle of the surgical capsule and hyperplastic glands, so the hyperplastic tissue was detached from the capsule as the complete gland without breakage $[12,16]$. Therefore, we compared the efficacy and safety of PKEP and TURP in the management of BPH. As expected, we found that PKEP had many advantages over shorter OT, IUT, and HST. Furthermore, significant heavier RTW, GRR, less BL, BT, SH, CP, BNC, and US were found in the PKEP group than in the TURP group. Moreover, QOL score DV and $Q_{\max }$ were higher in the PKEP group than in the TURP group, and postoperative IPSS in the PKEP group was significantly lower than that in the TURP group. All these illustrate that PKEP may be safe and applicable to the management of BPH.

TURS is defined as dilution hyponatremia $(<125$ $\mathrm{mmol} / \mathrm{L}$ ) and hypervolemia caused by rapid and massive absorption of irrigation fluid, combined with clinical cardiovascular dysfunction. Studies reported that the morbidity of TURS during TURP was $1.4 \%$, and the death rate was $0.1 \%$ [17]. Besides, the incidence rate increases when the resected tissue is heavier [18]. In the study, the incidence rate of TURS was 3\% in the TURP group, and no
TURS occurred in the PKEP group. It could be explained by the reason that surgeons needed to deal with the bleeding point after the tissue was resected layer by layer in the TURP group, which might lead to prolonged surgery time and increased absorption of irrigation fluid and relative high incidence of TURS. The median volume of prostate of patients in the modified PKEP group was not significantly bigger than that of the TURP group; however, OT in the PKEP group was significantly shorter than that in the TURP group. In the study, there was no statistical difference in prostate volume before surgery, and thus, our data indicated that shorter surgery time was needed for the performing of PKEP in patients with massive prostate, which was consistent with the other studies [19-21]. This means PKEP needed less time than TURP in treating large prostates. So, PKEP can reduce complications caused by long OT.

PKEP is the technology modified based on TURP, and the surgical technology (PKEP) has less impact on surrounding tissue than traditional surgical methods. So, PKEP can reduce the ONR, BS, CP, and RE. Some patients regarded their sexual function gets weakened after surgery in a previous publication [22]. The study focused on the occurrence rates of RE and erectile dysfunction and concluded that RE was common in patients treated by TURP. The incidence rate was significantly higher than that in the PKEP group [22, 23]. On the contrary, some patients in another study thought their erectile function, which was measured by the IIEF questionnaire, was unchanged after the surgery [19]. As a result, researchers concluded that PKEP did not have a negative impact on the postoperative erectile function [19]. In the study, there were no statistical differences between preoperative and postoperative IIEF-5, EEF, TAT, and STAT in the PKEP and TURP groups. Therefore, further researches are needed to determine whether PKEP has a negative effect on sexual function. The incidence of a multitude of complications in the PKEP group was lower than that in the TURP group, except TUI and RE. However, due to the small sample size, there was no significant statistical difference between the TURP group and the PKEP group.

PKEP was shown to have better safety than TURP. During the progression of PKEP, mirror sheath is similar to the surgeon's index finger in open surgery, which removed the gland under the direct view of the surgical capsule from the verumontanum to the bladder neck level [24]. After the gland was detached from the surgical capsule, the blood supply was cut off and hemostasis was implemented [25]. For this reason, the resection is nearly bloodless. Conversely, the hyperplastic tissue was resect- 
ed layer by layer in the TURP group, so the bleeding point needs to be dealt with after each resection. Thus, scabbing off and reopening of blood vessels may cause hemorrhage. Complications such as BL, postoperative BTs, and $\mathrm{SH}$ in the PKEP group are less likely to happen than that in the TURP group. Lower occurrence of the abovementioned complications in the PKEP group manifested its advantage over the traditional TURP. PKEP can remove hyperplastic tissue with intact surgical capsule, which decreases transfusion, indwelling catheter, BFT, and hospitalization time [26]. Another literature indicated that BT and clot retention were mostly observed in TURP among fifteen procedures applied to treat $\mathrm{BPH}$ [23]. The direct view of the surgical capsule and effective blockage of the arteriovenous trunk and punctiform hemorrhage between the capsule and hyperplastic gland contributed to the strong hemostatic capability of PKEP, which reduced intraoperative and postoperative bleeding.

PKEP can detach the hyperplastic prostate from the surgical capsule as a complete gland, and consequently, it may improve the integrity of the external urethral sphincter and bladder neck $[19,22]$. There was no significant difference in preoperative prostate volume compared to that in the TURP group. However, the postoperative prostatic volume was significantly different. It indicated that PKEP can remove more tissue than TURP. A publication showed that the removed tissue in the enucleation group had an average weight of $56.4 \mathrm{~g}$, and the elimination rate reached $81.2 \%$ [27]. By contrast, the average weight of resected tissue was $43.8 \mathrm{~g}$ in the resection group, and the resection rate was only $64.9 \%$ [27]. The current study found that the average weight of the enucleated gland was $73.1 \mathrm{~g}$ in the PKEP group, and the elimination rate in the PKEP group was $74.5 \%$ and that in the TURP group was $57.7 \%$. Adequate glandular ablation greatly improved the expected outcome after surgery and reduced the incidence associated with postoperative recurrence. A literature showed that the patients in the PKEP group had less lower urinary tract symptoms and more improvement in the QOL than patients in the TURP group in the postoperative follow-up [11]. In addition, no recurrent case was found during the 2-year follow-ups [11]. In our study, patients in the PKEP group had their IPSS dropped more than the TURP group postoperatively; nevertheless, there was no statistical difference. The PKEP group had significant improvement in QOL. All these results showed that PKEP had advantages in treating BPH in terms of efficacy and the prevention of recurrence [27]. Some indicators may have an impact on the efficiency of surgery, such as age, serum PSA, volume of prostate, preoperative IPSS and QOL, intravesical prostatic protrusion, and metabolic syndrome [28]. In the study, age, serum PSA, volume of prostate, preoperative IPSS, and QOL have no significant statistical difference between the TURP group and the PKEP group. Therefore, the impact of these indicators on the efficiency of surgery is negligible.

In the study, some clinical indicators in the PKEP group were similar to that of the TURP group, some were better than that of the TURP group, and the incidence of major complications in the PKEP group was less than that of the TURP group, which is consistent with the above PKEP advantages. In summary, PKEP and TURP have similar clinical efficacy in the treatment of massive $\mathrm{BPH}$. PKEP has advantages such as shorter OT, less BL, more GRR, and fewer complications, which may render it an efficient and safe alternative surgical way in the management of BPH. The research is limited by the number of enrolled patients as data were collected from a single center. Accordingly, longer follow-up, larger sample size, and more data collection from multiple centers are required to validate these results.

\section{Acknowledgements}

We would like to thank Beijing Geriatric Hospital, Beijing, China; Beijing Luhe Hospital, Capital Medical University, Beijing, China; Beijing TongRen Hospital, Capital Medical University, Beijing, China; and the Affiliated Hospital of Hebei, Baoding, Hebei, China.

\section{Statement of Ethics}

The protocol was approved by the Clinical Trial Ethics Committee at Beijing Luhe Hospital, Capital Medical University; Department of Urology, Beijing TongRen Hospital, Capital Medical University, Beijing, China; Department of Urology, the Affiliated Hospital of Hebei University, Baoding, Hebei, China; and Department of Urology, Beijing Geriatric Hospital, China. The patients were obtained from the above hospitals. Written informed consent was obtained from all patients or next of kin.

\section{Conflict of Interest Statement}

There are no other potential conflicts or relevant competing interests. All authors have no financial relationship with the organization that sponsored the research. The authors have full control of all primary data and agree to allow the journal to review our data if requested. The authors have nothing to disclose.

\section{Funding Sources}

The authors did not receive any funding. 


\section{Author Contributions}

Jian Zhang had full access to all the data in the study and takes responsibility for the integrity of the data and the accuracy of the data analysis. Study concept and design: Jian Zhang, Shujin Cui, and Yuexin Liu. Acquisition of data: Jian Zhang, Shuang Li, and Chunli Zhao. Analysis and interpretation of data: Jian Zhang, Yonghui Wang, ShiPeng Jin, Wenzeng Yang, and Yuexin Liu.
Drafting of the manuscript: Zhang Jian, Shuang Li, and Yonghui Wang. Critical revision of the manuscript for important intellectual content: Jian Zhang, Chunli Zhao, Wenzeng Yang, Shujin Cui, and Yuexin Liu. Statistical analysis: Jian Zhang, Shuang Li, Shiqing Zhang, Yonghui Wang, and ShiPeng Jin. Administrative, technical, or material support: Zhang Jian and Yuexin Liu. Supervision: Zhang Jian. Other: None.

\section{References}

1 Chughtai B, Forde JC, Thomas DD, Laor L, Hossack T, Woo HH, et al. Benign prostatic hyperplasia. Nat Rev Dis Primers. 2016;2: 16031.

2 Kim EH, Larson JA, Andriole GL. Management of benign prostatic hyperplasia. Annu Rev Med. 2016;67:137.

3 Namdarian B, Willder S, Steele G, Leona R, Grills R. Establishment of a urology service in a developing country: an observational study of outcomes in transurethral prostate resection procedures in Vanuatu. Lancet. 2015; 385(Suppl 2):S26.

4 Li Z, Chen P, Wang J, Mao Q, Xiang H, Wang $\mathrm{X}$, et al. The impact of surgical treatments for lower urinary tract symptoms/benign prostatic hyperplasia on male erectile function: a systematic review and network meta-analysis. Medicine. 2016;95(24):e3862.

5 Zou Z, Xu A, Zheng S, Chen B, Xu Y, Li H, et al. Dual-centre randomized-controlled trial comparing transurethral endoscopic enucleation of the prostate using diode laser vs. bipolar plasmakinetic for the treatment of LUTS secondary of benign prostate obstruction: 1-year follow-up results. World J Urol. 2018;36(7):1117.

6 Gudmundsson J, Sigurdsson JK, Stefansdottir L, Agnarsson BA, Isaksson HJ, Stefansson $\mathrm{OA}$, et al. Genome-wide associations for benign prostatic hyperplasia reveal a genetic correlation with serum levels of PSA. Nat Commun. 2018;9(1):4568.

7 Cha JY, Wee J, Jung J, Jang Y, Lee B, Hong GS, et al. Anoctamin 1 (TMEM16A) is essential for testosterone-induced prostate hyperplasia. Proc Natl Acad Sci U S A. 2015;112(31): 9722.

8 Bosch RJ. Pathogenesis of benign prostatic hyperplasia. Eur Urol. 1991;20(Suppl 1):27.

9 Xu D, Chen P, Xiao H, Wang X, DiSanto ME, Zhang X. Upregulated interleukin 21 receptor enhances proliferation and epithelial-mesenchymal transition process in benign prostatic hyperplasia. Front Endocrinol. 2019;10:4.

10 Suskind AM, Walter LC, Zhao S, Finlayson E. Functional outcomes after transurethral resection of the prostate in nursing home residents. J Am Geriatr Soc. 2017;65(4):699.
11 Xu P, Xu A, Chen B, Zheng S, Xu Y, Li H, et al. Bipolar transurethral enucleation and resection of the prostate: whether it is ready to supersede TURP? Asian J Urol. 2018;5(1):48.

12 Wu G, Hong Z, Li C, Bian C, Huang S, Wu D. A comparative study of diode laser and plasmakinetic in transurethral enucleation of the prostate for treating large volume benign prostatic hyperplasia: a randomized clinical trial with 12-month follow-up. Lasers Med Sci. 2016;31(4):599.

13 Feng L, Song J, Zhang D, Tian Y. Evaluation of the learning curve for transurethral plasmakinetic enucleation and resection of prostate using a mentor-based approach. Int Braz J Urol. 2017;43(2):245.

14 Gilling PJ, Aho TF, Frampton CM, King CJ, Fraundorfer MR. Holmium laser enucleation of the prostate: results at 6 years. Eur Urol. 2008;53(4):744.

$15 \mathrm{Xu} \mathrm{H}, \mathrm{Gu} \mathrm{M}$, Chen Q, Chen YB, Wang Z. [Modified morcellation procedure in holmium laser enucleation of the prostate]. Zhonghua Nan Ke Xue. 2016;22(11):991.

16 Luo YH, Shen JH, Guan RY, Li H, Wang J. Plasmakinetic enucleation of the prostate vs plasmakinetic resection of the prostate for benign prostatic hyperplasia: comparison of outcomes according to prostate size in $310 \mathrm{pa}$ tients. Urology. 2014;84(4):904.

17 Liu Z, Li YW, Wu WR, Lu Q. Long-term clinical efficacy and safety profile of transurethral resection of prostate versus plasmakinetic resection of the prostate for benign prostatic hyperplasia. Urology. 2017;103:198.

18 Reich O, Gratzke C, Bachmann A, Seitz M, Schlenker B, Hermanek P, et al. Morbidity, mortality and early outcome of transurethral resection of the prostate: a prospective multicenter evaluation of 10,654 patients. J Urol. 2008;180(1):246

19 Zhao Z, Ma W, Xuan X, Ou L, Liang Y, Zeng G. Impact of plasmakinetic enucleation of the prostate (PKEP) on sexual function: results of a prospective trial. J Sex Med. 2012;9(5):1473.
20 Peng M, Yi L, Wang Y. Photoselective vaporization of the prostate vs plasmakinetic resection of the prostate: a randomized prospective trial with 12-month follow-up in Mainland China. Urology. 2016;87:161.

21 Hirik E, Bozkurt A, Karabakan M, Aydemir H, Aktas BK, Nuhoglu B. Safety and efficacy of bipolar versus monopolar transurethral resection of the prostate: a comparative study. Urol J. 2015;12(6):2452.

22 Borchert A, Leavitt DA. A review of male sexual health and dysfunction following surgical treatment for benign prostatic hyperplasia and lower urinary tract symptoms. Curr Urol Rep. 2018;19(8):66.

23 Sun F, Sun X, Shi Q, Zhai Y. Transurethral procedures in the treatment of benign prostatic hyperplasia: a systematic review and meta-analysis of effectiveness and complications. Medicine. 2018;97(51):e13360.

24 Gilling P, Barber N, Bidair M, Anderson P, Sutton M, Aho T, et al. WATER: a doubleblind, randomized, controlled trial of aquablation(R) vs transurethral resection of the prostate in benign prostatic hyperplasia. J Urol. 2018;199:1252.

25 Liu C, Zheng S, Li H, Xu K. Transurethral enucleation and resection of prostate in patients with benign prostatic hyperplasia by plasma kinetics. J Urol. 2010;184(6):2440.

26 Chen S, Zhu L, Cai J, Zheng Z, Ge R, Wu M, et al. Plasmakinetic enucleation of the prostate compared with open prostatectomy for prostates larger than 100 grams: a randomized noninferiority controlled trial with long-term results at 6 years. Eur Urol. 2014;66(2):284

27 Zhao Z, Zeng G, Zhong W, Mai Z, Zeng S, Tao $\mathrm{X}$. A prospective, randomised trial comparing plasmakinetic enucleation to standard transurethral resection of the prostate for symptomatic benign prostatic hyperplasia: three-year follow-up results. Eur Urol. 2010;58(5):752.

28 Russo GI, Regis F, Spatafora P, Frizzi J, Urzì $\mathrm{D}$, Cimino S, et al. Association between metabolic syndrome and intravesical prostatic protrusion in patients with benign prostatic enlargement and lower urinary tract symptoms (MIPS Study). BJU Int. 2018;121:799. 